\title{
Identification of ZVS Points and Bounded Low-Loss Operating Regions in a Class-D Resonant Converter
}

Dahl, Nicolai Jerram; Ammar, Ahmed Morsi; Andersen, Michael A. E.

Published in:

IEEE Transactions on Power Electronics

Link to article, DOI:

10.1109/TPEL.2021.3052176

Publication date:

2021

Document Version

Peer reviewed version

Link back to DTU Orbit

Citation (APA):

Dahl, N. J., Ammar, A. M., \& Andersen, M. A. E. (2021). Identification of ZVS Points and Bounded Low-Loss Operating Regions in a Class-D Resonant Converter. IEEE Transactions on Power Electronics, 36(8), 9511 9520. https://doi.org/10.1109/TPEL.2021.3052176

\section{General rights}

Copyright and moral rights for the publications made accessible in the public portal are retained by the authors and/or other copyright owners and it is a condition of accessing publications that users recognise and abide by the legal requirements associated with these rights.

- Users may download and print one copy of any publication from the public portal for the purpose of private study or research.

- You may not further distribute the material or use it for any profit-making activity or commercial gain

- You may freely distribute the URL identifying the publication in the public portal 


\title{
Identification of ZVS Points and Bounded Low-Loss Operating Regions in a Class-D Resonant Converter
}

\author{
Nicolai J. Dahl, Student Member, IEEE, Ahmed M. Ammar, Member, IEEE, \\ Michael A.E. Andersen, Member, IEEE
}

\begin{abstract}
This paper presents an analysis of the different loss modes of the switching devices in a class-D series resonant converter operating with either fixed dead time or fixed duty cycle. A feasible operating region where the FETs in the inverter stage only exhibit reverse conduction losses, with no hard-switching, is identified. Furthermore, the impact of using a fixed dead time versus a fixed duty cycle is investigated. We find that using a fixed dead time is superior to using a fixed duty cycle, as a broader operating range can be achieved for the same losses, or the same operating range can be achieved with lower losses. A reduction in the reverse conduction losses of up to $59 \%$ or an expansion of the operational frequency range by $33 \%$ when using a fixed dead time is found. The modeling approach is validated on a 1-MHz prototype employing GaN switching devices. Lastly, a design example shows how the presented analysis can be used to determine the optimal fixed dead time/duty cycle for use with frequency modulation control such that the losses are minimized.
\end{abstract}

Index Terms-Resonant converters, Modeling, First Harmonic Approximation, DC-DC power converters, Wide-Bandgap Devices.

\section{INTRODUCTION}

$\mathbf{T}$ ODAY, most electronic devices are powered with a Switch Mode Power Supply (SMPS) thanks to their small form factor and high efficiency. The majority of SMPS are different types of hard-switching PWM topologies. These supplies have high switching losses, which limit the switching frequency that the supplies can handle. Nonetheless, higher switching frequencies allow for smaller passive components, and hence an overall smaller power supply and higher bandwidth to better react to various load and line disturbances. To improve the switching frequencies, soft-switching topologies have received much attention in recent years [1-7]. One family of soft-switching converters is the resonant converters [8]. Resonant converters work by having a resonant tank responsible for providing AC gain, in addition to charging and discharging the switching node during the dead time before the switching event and thus obtain Zero-Voltage Switching (ZVS). This vastly reduces the switching losses, allowing for a higher operating frequency.

A popular family of resonant converter is the class-D series resonant converter [9-12] and its derivatives like the LLC

This project has received funding from the European Union's Horizon 2020 research and innovation programme under grant agreement No. 731466. The authors are with the Department of Electrical Engineering, Technical University of Denmark, Kgs. Lyngby (e-mail: nicoje@elektro.dtu.dk; ammma@elektro.dtu.dk; ma@elektro.dtu.dk)
[13-17]. These converters benefit from low device stress compared to other typologies like the class-E [4, 18, 19] and have a broad range of applicable controllers available. Some of the most common control techniques are: Tunable resonant tank capacitor control [20-23], bang-bang control [24-27], frequency modulation (FM) control [28-31], and lastly, phase-shift control $[32,33]$. Unlike any of the other control methods, the phase-shift control uses double the number of switches in the inverter to achieve phase-control, thereby making it less compelling if cost or power density is of concern. In many cases, it is of interest to control the output voltage of the resonant converter, usually to compensate for changes or disturbances in the load or the supply voltage. Besides controlling the output voltage, it is paramount that the resonant converter upholds ZVS, which complicates the control design. These two simultaneous objectives can often be met using phase-shift control or combining FM-control with either a tunable resonant tank capacitor or adaptive duty cycle/dead time scheme [34]. However, using phase-shift control or some combined control methods vastly increase the complexity of the control circuit. As a simple control strategy is normally preferred, frequency control is often applied. Hence it is of interest to know the exact range of operation before ZVS is lost.

The investigation and analysis of ZVS operation for resonant converter has been widely covered in prior art. However, the coverage usually focuses on the converter design process to obtain ZVS and is not concerned with post-design considerations like the operational range and whether using a fixed duty cycle or fixed dead time is superior over the operational frequency range. In [9], the class-D series resonant converter is analyzed. The analysis only considers the use of fixed duty cycle for the dead time generation, leaving out the fixed dead time option. This is also the case for $[10,11,13,18,19,33]$.

This paper presents an analysis of the different switching loss modes in the GaNFETs for a class-D Series Resonant Converter (SRC) when the duty cycle/dead time is assumed fixed and FM-control is utilized. A complete mapping of the converter behavior is presented. Here, multiple ZVS points for the same dead time and limitations of the operating range are identified. Further, we define a feasible soft-switching operation region that expands the operating space previously determined in [9]. In the feasible region, we determine the evolution of the losses and define an upper loss bound for the GaNFETs. The analysis considers the case where a fixed 
dead time is used and the case where a fixed duty cycle is used and determines the best of the two options. The findings in this paper can be used for determining the optimal fixed dead time/duty cycle for use with FM-control while also providing valuable information for both the design of the converter and selection of switching devices. The analysis is validated on a 1-MHz class-D SRC, where the feasible region and the location of the worst-case losses within the region are measured. Finally, a design example is provided on how to use the analysis for control design.

Although the presented analysis and results are carried out for a class-D SRC, the analysis and findings are applicable for any resonant converter that uses a sinusoidal current to charge and discharge the switching node. Such converters are other class-D based converters using either parallel and/or series resonance tanks like the LLC and LCC converters.

\section{LOSSES IN INVERTER SWITCHING DEVICES}

Fig. 1 shows a Class-D SRC with the parasitic capacitances for the GaNFETs $C_{o s s_{H}}$ and $C_{o s s_{L}}$. It is assumed that the converter operates above the resonance frequency in the inductive mode, where the resonant current lags the switching node voltage, and that the parasitic capacitances are static. When driving the converter outside the point of ZVS, losses occur in the FETs either due to insufficient charging/discharging of the combined output capacitance $C_{o s s_{(t)}}$, reverse conduction losses of the FETs, or both. This section presents the loss mechanisms with the magnitude and conditions for each type of switching loss.

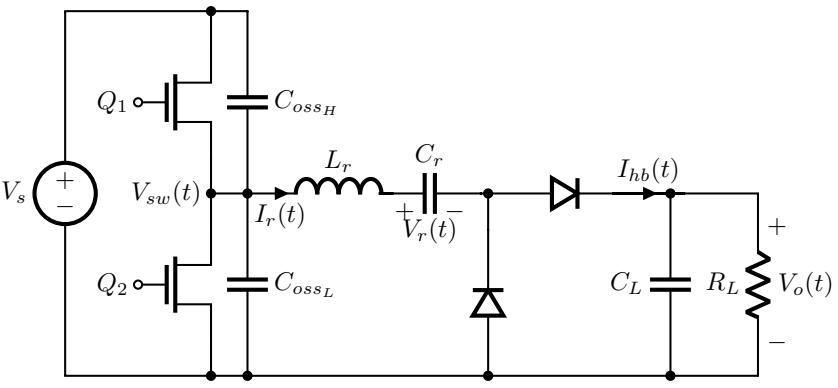

Fig. 1: The class-D series resonant converter with the parasitic capacitance for the FETs.

For resonant converters, ZVS is achieved by having the resonant tank current $I_{r}$ charge/discharge the switching node $V_{s w}$ to $V_{s}$ /ground before turning on the corresponding FET. Doing this results in zero volts across the FET at the switching moment, thus achieving ZVS, and no power is dissipated. Due to the symmetry of the resonant current and the power stage, achieving ZVS in the charge direction implies that ZVS is likewise achieved in the discharge direction. Hence the presented math in this and the following sections will focus on the charging case only when determining ZVS and losses. Also, the time dependency is omitted from the equations.

The time that $I_{r}$ has to charge $C_{o s s_{(t)}}$ to $V_{s}$ is given by the dead time $t_{d}$, which is related to the duty cycle by:

$$
t_{d}=\frac{0.5-D}{f_{s w}}
$$

where $D$ is the duty cycle for each FET and $f_{s w}$ is the switching frequency of the converter. In some cases, the dead time is implemented as a constant time and independent of the switching frequency. If the dead time is too short or the resonant current too small, $C_{o s s_{(t)}}$ is insufficiently charged, leading to a voltage across the FET at the switching moment that will result in switching loss. Equation (2) shows the loss. $V_{D S}$ is the voltage across the FET at the switching moment.

$$
P_{s w}=\frac{1}{2} C_{o s s(t)} V_{D S}^{2} f_{s w}
$$

If the dead time is too long, the GaNFET will enter reverse conduction, which prevents overcharging of $C_{\text {oss }}$ but leads to reverse conduction losses. The voltage drop across GaNFETs in reverse conduction, $V_{f}$, tend to be higher than that for MOSFETs. However, since GaN switches have zero reverserecovery charge $\left(Q_{r r}=0\right)$, unlike MOSFETs, the reverse recovery losses become zero, leaving the reverse conduction loss to be the only loss of interest. For applications where the dead time is short, the $\mathrm{GaN}$ device is more efficient than a MOSFET from a reverse conduction point of view. Equation (3) shows the reverse conduction loss. $V_{f}$ is the voltage drop across the FET in reverse conduction, and $t_{\text {cond }}$ is the reverse conduction time.

$$
P_{\text {rev }}=V_{f} t_{\text {cond }} I_{r} f_{s w}
$$

Lastly, there are the two cases where the dead time is so long that the resonant tank current changes direction at time $t_{r}<t_{d}$ causing an undesired discharge of the switching node $V_{s w}$. In the first case, $V_{s w}$ is completely charged hence both reverse conduction losses and switching losses occur. In the second case, $V_{s w}$ is insufficiently charged, resulting in switching losses. Fig. 2 shows the five types of switching with the corresponding resonant tank current. From a loss modeling perspective, the first and fifth case are equivalent.

Through a rewriting of (2) and (3), the losses of the four lossy switching modes in Fig. 2 can be determined. In the first and fifth case where partial hard-switching losses occur, $V_{D S}$ can be determined as the voltage over $C_{o s s_{(t)}}$ due to the accumulated charge from $I_{r}$ during the dead time subtracted from the supply voltage. Hence (2) can be rewritten to (4).

$$
P_{s w}=\frac{1}{2} C_{o s s(t)} f_{s w}\left(\frac{1}{C_{o s s_{(t)}}} \int_{t_{d}} I_{r} d t-V_{s}\right)^{2}
$$

In the third case where reverse conduction losses occur, the term $t_{\text {cond }} I_{r}$ provides the number of charges passing through the GaNFET during the conduction time. This can be written as the total number of charges accumulated during the dead time subtracted with the number of charges needed to charge $C_{o s s_{(t)}}$ to $V_{s}$. Equation (5) shows the rewritten version of (3).

$$
P_{r e v}=V_{f} f_{s w}\left(\int_{t_{d}} I_{r} d t-V_{s} C_{o s s_{(t)}}\right)
$$



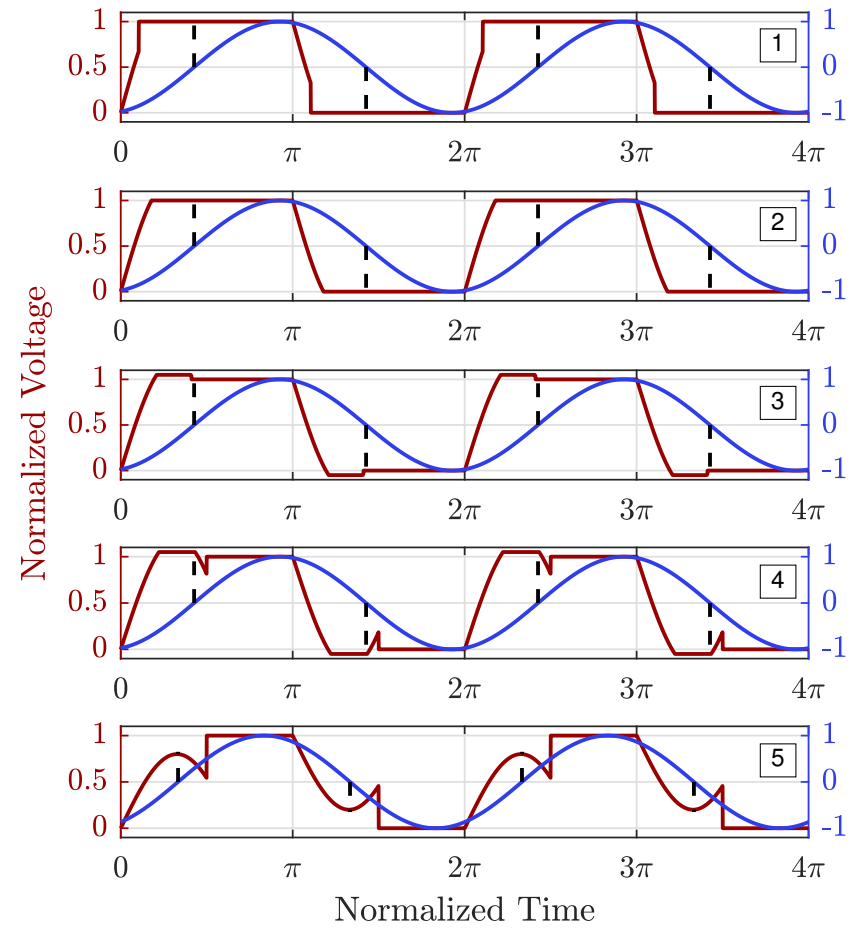

Fig. 2: Typical switching waveforms for $V_{s w}(t)$ and resonant tank currents $I_{r}(t)$. The dashed lines indicate the zerocrossing of the current. From top to bottom: Partial hardswitching; where the dead time is too short resulting in a jump in the voltage at turn on/off. $Z V S$; where $V_{s w}(t)$ is fully charge/discharged during the dead time. Reverse conduction; where reverse conduction occurs due to long dead time. Current reversal; where $V_{s w}(t)$ starts to discharge again before the end of the dead time. Partial hard switched current reversal; where the dead time is too long and the current too small resulting in an increased jump in the voltage at turn on/off compared to case 1 .

Finally, in the fourth case, where both reverse conduction losses and switching losses occur due to a long dead time causing the reversal of the current flow, the losses can be written as in (6). Here the charges for $P_{r e v}$ are only accumulated up to the point where the current flow changes direction $\left(t_{r}\right)$, and the switching loss is evaluated from $t_{r}$ to the end of the dead time $t_{d}$.

$$
\begin{array}{r}
P_{r e v}=V_{f} f_{s w}\left(\int_{t_{r}} I_{r} d t-V_{s} C_{o s s_{(t)}}\right) \\
P_{s w}=\frac{1}{2} C_{o s s_{(t)}} f_{s w}\left(\frac{1}{C_{o s s_{(t)}}} \int_{t_{d}-t_{r}} I_{r} d t\right)^{2}
\end{array}
$$

\section{SWitching Loss AnALYsis}

In this section, we determine a feasible operation region with regards to power losses. The region is encircled by the ZVS points and the boundary where the resonant current changes direction. Thus, both of these behaviors will be described analytically. Furthermore, the losses induced in the
FET's related to switching are determined for the feasible region. The analysis is carried out using the First Harmonic Approximation (FHA) approach [8] combined with the methods presented by Hamill in [35] to include the effects of the parasitic capacitance in the rectifier diodes. In the analysis, the frequency is normalized by the resonance frequency to generalize the findings.

Using the FHA, we assume that the resonance current, $I_{r}$, can be adequately described as a pure sine waveform. Equation (7) shows the approximated resonance current $\hat{I}_{r}$.

$$
I_{r} \approx \hat{I}_{r}=I_{m} \sin \left(\omega_{n} t+\phi\right)
$$

where $I_{m}$ is the magnitude of the current given by (8a), and $\phi$ is the phase-shift between the resonant current and $V_{s w}$ given by $(8 b)$.

$$
\begin{aligned}
I_{m} & =\frac{\left|\mathcal{F}_{1}\left\langle V_{s w}\right\rangle\right|}{R_{e q} \sqrt{1+Q^{2}\left(\omega_{n}-\omega_{n}^{-1}\right)^{2}}} \\
\phi & =-\arctan \left(Q\left(\omega_{n}-\omega_{n}^{-1}\right)\right)
\end{aligned}
$$

$\mathcal{F}_{n}\langle\cdot\rangle$ denotes the function for the $n$ 'th harmonic Fourier series, and $\omega_{n}$ is the normalized angular switching frequency given by:

$$
\omega_{n}=\omega / \omega_{c}
$$

where $\omega$ is the switching frequency and $\omega_{c}$ is the resonance frequency of the converter in rad/sec. Lastly, $Q$ is the resonant tank quality factor, and $R_{e q}$ is the ideal equivalent resistive load of the rectifier as seen from the output of the inverter and is given by (10).

$$
R_{e q}=\frac{2 R_{L}}{\left(\pi+C_{d} \omega R_{L}\right)^{2}}+R_{e s r}
$$

where $R_{L}$ is the load resistance, $C_{d}$ is the parasitic capacitance of the rectifier diode, and $R_{e s r}$ is the combined parasitic series resistance in the resonant tank. In Hamill's work, the rectifier diodes' parasitic capacitance is modeled as a capacitor, $C_{h}$ in series with $R_{e q}$. The capacitor $C_{h}$ being in series with the resonant capacitor $C_{r}$ effectively reduces the total resonant tank capacitance and makes it dependent on the switching frequency. This impacts the resonance frequency, $\omega_{c}$, and the quality factor $Q$ such that both increase slightly with the switching frequency. Thus, $Q$ is found as:

$$
Q=\frac{C_{r}+C_{h}}{C_{r} C_{h} R_{e q} \omega_{c}}
$$

Modeling the small increase in both the resonance frequency and quality factor results in a better prediction of the resonant current when the switching frequency is far away from the resonance frequency of the converter, hence improving the fidelity of the model. 


\section{A. Zero-Voltage Switching Points}

Zero-Voltage Switching can be achieved whenever the accumulated charge from the resonant current is equal to that needed to charge the parasitic capacitance, $C_{o s s_{(t)}}$ to the supply voltage, $V_{s}$. The resonance current is only able to deliver charge during the dead time, which is assumed fixed. Hence to obtain ZVS, the equality in (12) needs to be true.

$$
\int_{\bar{t}_{z v s}}-\hat{I}_{r} d t-V_{s} \bar{C}_{o s s_{(t)}}=0
$$

Where $\bar{t}_{z v s}$ is the normalized dead time needed for ZVS when the frequency is $\omega_{n}$ and $\bar{C}_{o s s_{(t)}}$ is the normalized parasitic capacitance defined as:

$$
\bar{C}_{o s s_{(t)}}=C_{o s s_{(t)}} \omega_{c}
$$

Inserting (7) in the integral of (12) and evaluating the integral we get:

$$
\frac{I_{m}\left(\cos \left(\omega_{n} \bar{t}_{z v s}+\phi+\theta\right)-\cos (\phi+\theta)\right)}{\omega_{n}}-V_{s} \bar{C}_{o s s_{(t)}}=0
$$

where $\theta=\left\langle\mathcal{F}_{1}\left\langle V_{s w}\right\rangle\right.$. The switching node $V_{s w}$ is described by a piecewise function such that the charge and discharge of the output capacitance is included [28]. A result of this is that the phase of the Fourier approximation becomes non-zero. Thus $\theta$ accounts for any phase-shift present in $V_{s w}$ such that it aligns properly with $I_{r}$. By solving for $\bar{t}_{z v s}$ in (14), an expression for the dead time needed to obtain ZVS is found.

$$
\bar{t}_{z v s}=-\frac{\phi+\theta+\arccos \left(\cos (\phi+\theta)+\frac{V_{s} \omega_{n} \bar{C}_{o s s}(t)}{I_{m}}\right)}{\omega_{n}}
$$

To obtain a condition for ZVS similar to (15) but for a fixed duty cycle, the relation in (1) is used in its normalized form (16).

$$
\bar{t}_{z v s}=2 \pi \omega_{n}^{-1}\left(0.5-D_{z v s}\right)
$$

Substituting $\bar{t}_{z v s}$ with (15) and solving for the duty cycle results in (17).

$$
D_{z v s}=\frac{1}{2}+\frac{\phi+\theta+\arccos \left(\cos (\phi+\theta)+\frac{V_{s} \omega_{n} \bar{C}_{o s s}(t)}{I_{m}}\right)}{2 \pi}
$$

The results (15) and (17) are both, in theory, independent of $V_{s}$, meaning that ZVS will be achieved for any given supply voltage. However, in practice, $C_{o s s_{(t)}}$ changes with $V_{s}$ in a nonlinear manner, thereby moving the ZVS points slightly. The effect scales with $\omega_{n}$ and $R_{L}$ such that the ZVS points close to resonance move less than those far from resonance, and the movement is increased for smaller loads. Hence, the determined ZVS points from (15) and (17) are only valid when the supply voltage is within a local neighborhood of $V_{s}$.

By sweeping $\omega_{n}$ in (15) and (17) it is possible to trace out the ZVS curve. Fig. 3 shows the obtained ZVS curve for a fixed duty cycle (Fig. 3a) and a fixed dead time (Fig. 3b) when the resonant tank has a $Q=4$. As seen, the computed solution indicates that two simultaneous ZVS points exist for each value of the fixed dead time in the normalized range from 0.44 to 0.68 and fixed duty cycle values between $42.2 \%$ and $38.5 \%$. The phenomenon occurs because the resonant tank current $I_{r}$ changes in both amplitude and phase with frequency. For the close to resonance part of the ZVS curve, i.e. the ZVS curve below the "Max Loss" curve, the resonant current is large and almost in phase with the switching node. This results in ZVS being obtained primarily due to the large amplitude of the current. For the far from resonance part of the ZVS curve, i.e. the ZVS curve above the "Max Loss" curve, the current is smaller, and ZVS is achieved due to the increased phase-shift. At the very top and bottom of the feasible operating region where the ZVS curve intersects with the "Rev. Limit" curve, class-DE operation is achieved, as Zero-Voltage-derivative Switching (ZVdS) and thereby also Zero-Current Switching (ZCS) occur. The ZVdS results in a decreased sensitivity to changes in the dead time, which is seen in Fig. 3 by the small slope of both the low and high frequency ZVS. At large dead times or small duty cycles, the low frequency section of the ZVS curve disappears due to the reversal of the current flow marked by the dashed line.

\section{B. Current Flow Reversal Limit}

During the desired operation of a resonant converter, the resonance current will monotonically charge the switching node during the dead time period. However, if the dead time becomes too extensive, the resonant current's direction will change during the dead time, resulting in an unwanted discharge of the switching node near the end. This behavior inhibits ZVS. Thus it is of interest to work out when this phenomenon occur.

Whenever the current flow changes direction, a zero-crossing occurs. This means that the time needed to reach the zerocrossing is equivalent to finding the point of the current flow reversal. Hence we have:

$$
-I_{m}\left(\omega_{s}\right) \sin \left(\omega_{n} \bar{t}_{r}+\phi+\theta\right)=0
$$

Where $\bar{t}_{r}$ is the normalized time needed to reach the zerocrossing given by: $\bar{t}_{r}=t_{r} \omega_{c}$. The only practical solution to (18) is when the trigonometric term is zero. Due to the nature of the sine function, it follows:

$$
\begin{aligned}
& \Rightarrow-\omega_{n} \bar{t}_{r}-\phi-\theta=0 \\
& \Leftrightarrow \bar{t}_{r}=(\phi+\theta) / \omega_{n}
\end{aligned}
$$

From where the zero-crossing time is found. Using the relationship between the normalized time and duty cycle from (16), the corresponding duty cycle limit, $D_{r}$, can be determined:

$$
\begin{aligned}
& 2 \pi \omega_{n}^{-1}\left(0.5-D_{r}\right)=(\phi+\theta) / \omega_{n} \\
& \Leftrightarrow D_{r}=\frac{1}{2}-\frac{\phi+\theta}{2 \pi}
\end{aligned}
$$




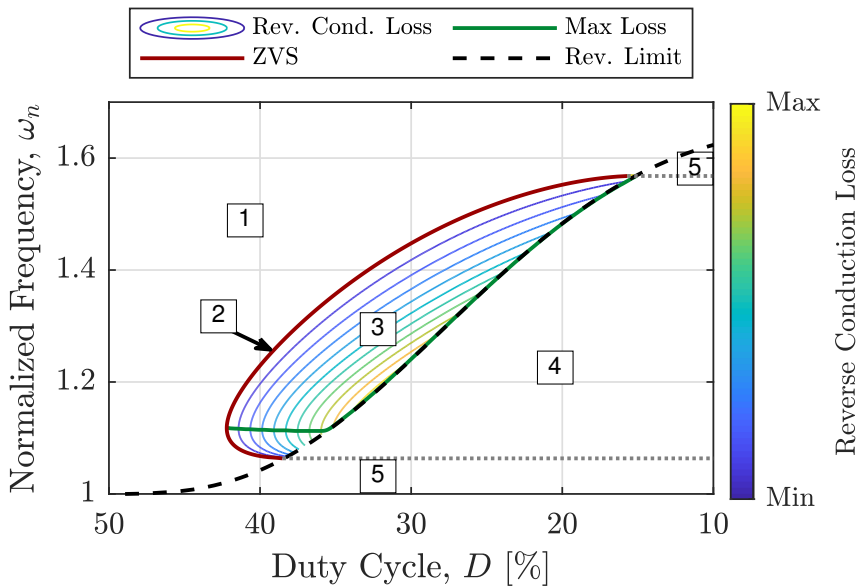

(a)

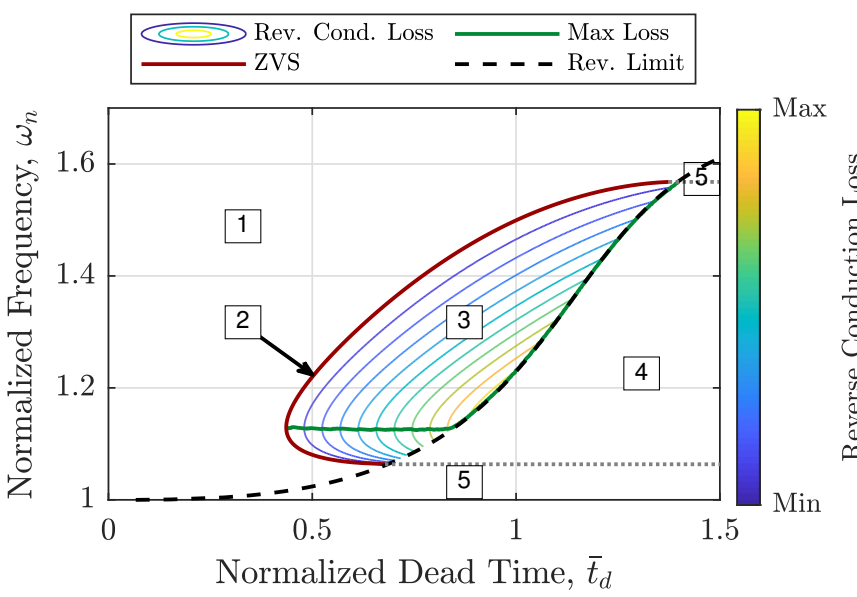

(b)

Fig. 3: The feasible operation region of the resonant converter encircled by the ZVS curve and the current reversal boundary. (a) shows the region when duty cycle is used, and (b) shows the region for a normalized dead time. The max loss curve indicates the point of the worst-case loss for every duty cycle/dead time inside the feasible region. The numbers indicate the different operating regions and match the numbering in Fig. 2.

The dashed lines in Fig. 3 show the boundaries for where the current flow reversal occurs. Fig. 3a shows it when operating with a fixed duty cycle (22), and Fig. 3b for when operating with a fixed dead time (20). Due to the nature of the current flow reversal limit, ZCS will always happen when operating on the limit.

\section{Maximum Reverse Conduction Loss}

The area encircled by the ZVS curve and the reverse current boundary in Fig. 3 is the region of reverse conduction losses. It is usually preferable to stay in this region compared to staying in the region with quadratic switching losses, since lower losses are achievable. This is especially the case with $\mathrm{GaN}$ devices thanks to their zero reverse recovery charge. Thus, the encircled region with its encirclement constitutes the feasible operating region for the resonant converter. As only reverse conduction losses occur in the feasible region, an upper bound of the losses in the FETs can be determined to assess the worst-case operation mode and location.

To find the worst-case reverse conduction losses in the feasible operating region, we first evaluate the losses in every point in the region using the normalized reverse conduction loss given by (24). Finally, the maximum loss for each dead time/duty cycle is logged.

$$
\begin{aligned}
\bar{P}_{\text {rev }} & =V_{f} \frac{\omega_{n}}{2 \pi}\left(\int_{\bar{t}_{d}}-I_{r} d t-\bar{C}_{o s s_{(t)}} V_{s}\right) \\
& =V_{f} \frac{\omega_{n}}{2 \pi} \int_{\bar{t}_{z v s}}^{\bar{t}_{d}}-I_{r} d t
\end{aligned}
$$

In Fig. 3, the contour lines indicate the magnitude of the reverse conduction losses in the feasible region. The curve "Max Loss" represents the point of the worst-case loss for every duty cycle/dead time inside the feasible region. The max loss curve follows the contour lines by intersecting the contours' peaks, which indicate the highest loss point for the given dead time/duty cycle. Once the max loss curve reaches the current flow reversal limit, the worst-case loss will follow the limit until it reaches the upper bound of the feasible region. Lastly, the curve for the current flow reversal boundary indicates the endpoints of where ZVS is achievable. However, increasing the dead time slightly beyond this boundary does not significantly impact the losses. This is because the reverse current flow reduces the voltage difference due to the forward voltage drop that would otherwise create an additional small switching loss.

\section{Converter Design Considerations}

Based on the found feasible region, some remarks can be added regarding how to design the converter to enhance the feasible region. A usable design metric is to look at the rotation of the feasible region. If the feasible region is rotated counter-clockwise, more switching frequencies will be available for the same dead time. This means that an FM-controller will achieve a larger span of output voltages at the same amount of losses or the same output span for fewer losses. The counter-clockwise rotation can be achieved by adjusting the quality factor $\mathrm{Q}$ and by adjusting the output capacitance of the FETS $C_{\text {oss }}$. By increasing Q, a larger phase-shift in the resonant tank will occur for the same frequency step, corresponding to a counter-clockwise rotation of the feasible region. Likewise, by decreasing $C_{o s s}$, less charge is needed to obtain ZVS. As a result, a rotation of the feasible region occurs, and the region shifts slightly to the left because less dead time is needed.

A high Q-factor is often used to ensure a high gain sensitivity, which guarantees the FHA, while a low $C_{\text {oss }}$ is usually picked to reduce the needed dead time and/or limit the losses during partial hard-switching. Thus the design recommendations for rotating the feasible region coincide with the typical design recommendations for class-D resonant converters. 


\section{Comparison of Dead Time and Duty Cycle}

For a selected dead time/duty cycle, the feasible operating region will have a certain range of switching frequencies where the converter will operate within the region. For a normalized dead time of 0.5 , the range of feasible operating frequencies is approximately 0.15 . However, as the dead time increases, the range expands until the effect of the current reversal becomes severe and begins limiting the range once again. Fig. 4 shows this effect for both the fixed dead time and the fixed duty cycle cases.

As the dead time is increased, the feasible operating frequency range expands until it reaches 0.31 from where it rapidly contracts due to the current reversal limiting the switching frequency from below. The same effect is observed when the duty cycle is decreased. However, the duty cycle is only able to reach a maximum frequency range of 0.23 . Moreover, the maximum losses in the feasible region when using a fixed duty cycle is higher than using a fixed dead time. More specifically, the analysis shows that a fixed dead time provides an expansion of up to $33 \%$ in the operating range for the same losses compared to using a fixed duty cycle, or up to a $59 \%$ decrease in reverse conduction losses for the same operating range compared to using a fixed duty cycle.

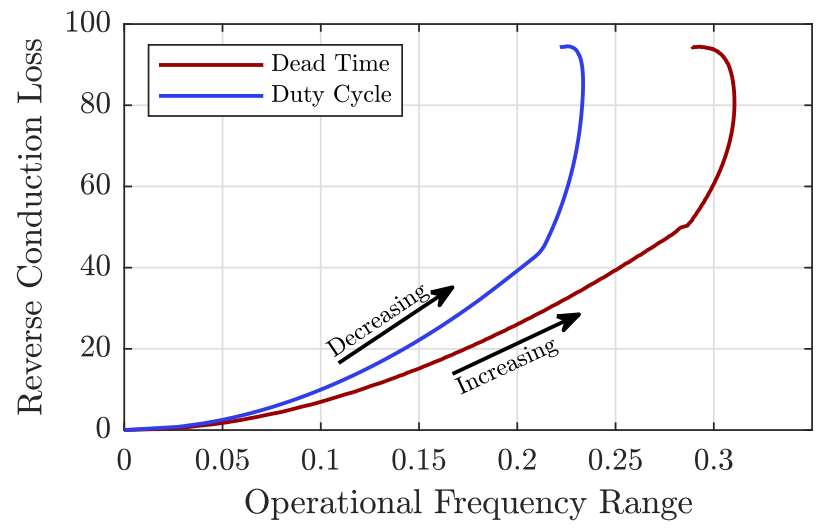

Fig. 4: The operational frequency range against the normalized worst-case reverse conduction loss in the feasible region for the given range. The arrows are indicating the direction of increased dead time and decreased duty cycle, equivalent of moving to the right on Fig. 3. The dead time and duty cycle each moves up to the point of the highest reverse conduction loss.

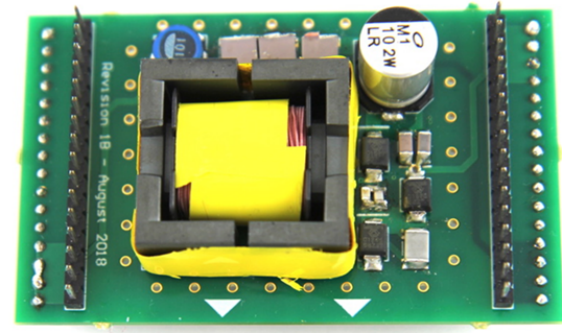

Fig. 5: Prototype class-D SRC.
TABLE I: Specifications of the converter. The capacitances, $V_{f}$, and $R_{f}$ are based on device characterization using a Keysight B1505A power device analyzer.

\begin{tabular}{c|cll}
\hline & Value & Unit & Acquired \\
\hline$V_{s}$ & 350 & $\mathrm{~V}$ & Measured \\
$R_{L}$ & 1000 & $\Omega$ & Measured \\
$R_{e s r}$ & 2 & $\Omega$ & Measured \\
$C_{o s s}(t r)$ & 40 & $\mathrm{pF}$ & Measured \\
$C_{d}$ & 12.6 & $\mathrm{pF}$ & Measured \\
$V_{f}$ & 1.3 & $\mathrm{~V}$ & Measured \\
$R_{f}$ & 0.6 & $\Omega$ & Measured \\
$f_{c}$ & 836 & $\mathrm{kHz}$ & Calculated \\
\hline
\end{tabular}

\section{EXPERIMENTAL VALIDATION}

To validate the analysis findings, a 1-MHz class-D resonant converter prototype using GaNFETs [36] for the inverter switches is constructed. A complete characterization is performed on both the GaNFETs and the rectifier diodes using a Keysight B1505A power device analyzer to obtain accurate data for the model. To characterize the output capacitance, the capacitance is measured for multiple bias voltages across the device going from $0 \mathrm{~V}$ to $V_{s}$. Then the average of the acquired capacitance values is calculated to get the time-related output capacitance $C_{o s s_{(t r)}}$. The same approach is used to obtain the rectifier diode capacitance $C_{d}$ with the only difference being that the bias voltage is limited to $250 \mathrm{~V}$. The reverse conduction characteristic of the GaNFET is modeled as a diode using a forward voltage $V_{f}$ and a resistor $R_{f}$. Fig. 5 shows the prototype and Table I shows the specifications and test conditions.

Points across both the ZVS curve and the current reversal curve are measured for encircling the feasible region and for comparison with the analysis. The measurements are performed by changing the converter's switching frequency and duty cycle until ZVS is observed. The same procedure is used for the current reversal. To determine the curve and size of the worst-case reverse conduction losses in the feasible region, an experiment is designed to map the response surface in the feasible region. Thus the reverse conduction losses are measured at multiple points inside the feasible region. A $4^{\text {th }}$ order model is fitted from the measurements, and the maximum loss is derived from the estimated model.

Fig. 6a shows the measurement results (points) with the expected results from the analysis (lines) for a fixed duty cycle, and Fig. $6 \mathrm{~b}$ shows the measurement results for the fixed dead time. Looking at Fig. 6, we find that the ZVS measurements and the location of the worst-case losses correspond to the theory. The ZVS measurements follow the theoretical curve exactly until $1.2 \mathrm{MHz}$, where the measurements start to fall slightly below the curve while the max loss points are measured to be at a slightly higher frequency than predicted. The reverse current limit follows the expected curve up to approximately $1 \mathrm{MHz}$ from where the points are found at a greater dead time than expected. The discrepancy between the measurement and theory with the 


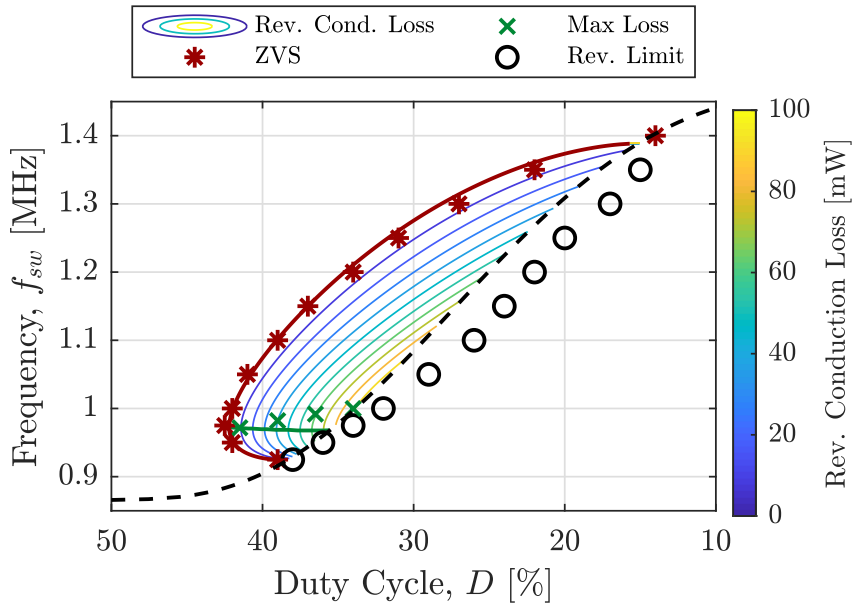

(a)

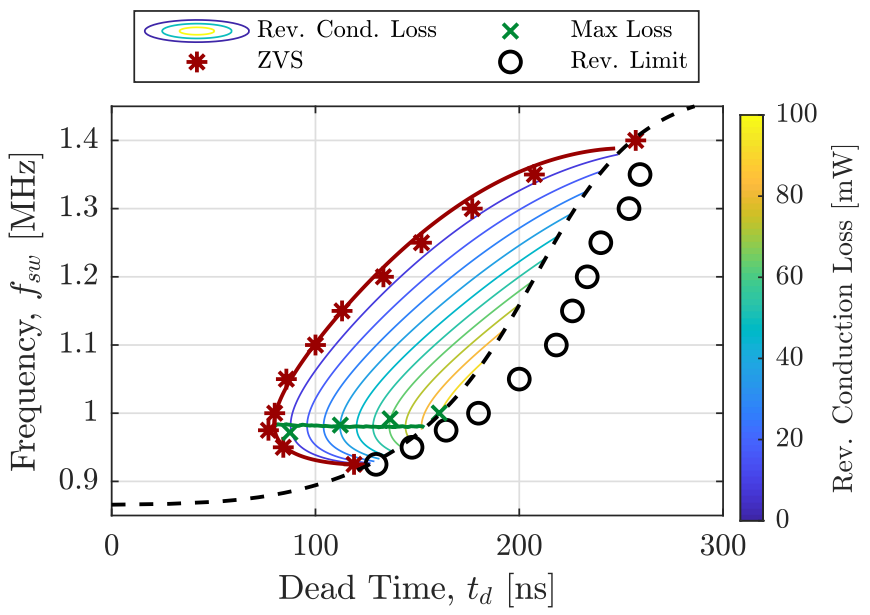

(b)

Fig. 6: Measured (points) and analytic (lines) results for the prototype converter. (a) shows the result for the fixed duty cycle, and (b) shows the results for the fixed dead time.

increasing switching frequency is partly due to the breakdown of the FHA method since the resonant current $I_{r}$ no longer acts like a pure sinusoidal current. Furthermore, at higher switching frequencies, the model becomes more sensitive to the parasitic capacitance values in the GaNFETs and rectifier diodes.

The measured feasible region, both when using a fixed dead time and a fixed duty cycle, assimilates the shape of the corresponding theoretical region, with the main difference being the lower reverse current limit that expands the region slightly more. This suggests that the difference in performance identified in section IV is valid and a fixed dead time in general is preferred.

Fig. 7 shows eight scope shots taken from the prototype when operating in the different regions as marked in Fig. 3. Fig. $7 \mathrm{a}$ and $7 \mathrm{~b}$ show the class-DE operation at the very top and bottom of the feasible region respectably. Fig. $7 \mathrm{~b}$ and $7 \mathrm{~d}$ show ZVS for the same duty cycle but at two different frequencies proving that there can exist two ZVS points for the same duty cycle/dead time. Fig. $7 \mathrm{c}$ to $7 \mathrm{f}$ show the switching behavior for region 1 through 4 . Finally, Fig. $7 \mathrm{~g}$ and $7 \mathrm{~h}$ show the switching behavior of region 5 at the low and high frequency part respectively.

From Fig. 7a and $7 \mathrm{~b}$ it is clear to see that $V_{s w}$ charges up with a decreasing slope and reaches the final voltage with almost zero slope, indicating that the resonant current is zero i.e. ZCS is achieved, which implies class-DE operation. Lastly, we find that all the waveforms for all the tested operating points behave as expected from the analysis and match with the expected waveforms for each region provided in Fig. 2.

\section{Design ExAMPLE}

In this last section, an example is provided to show how the presented analysis can be applied in practice. For the example, the same prototype class-D SRC as in section V is used, and we assume that it operates with a fixed dead time and frequency control. The design objective is to keep a stable output voltage, $V_{o}$, for a changing supply voltage $V_{s}$ and load $R_{L}$. This needs to be achieved while staying within the feasible operating region at all time to minimize losses in the switching devices. The maximum variation on the supply voltage and load is assumed to be bounded. Table II shows the design criteria. The design procedure can be summarized in three steps:

1) Determine the lowest and highest switching frequency $\left(f_{s w_{\min }} \& f_{s w_{\max }}\right)$ needed to stay at $V_{o}$ for all possible perturbations.

2) Derive the feasible operating regions for the perturbations resulting in $f_{s w_{\min }}$ and $f_{s w_{\max }}$.

3) Find a common dead time that keeps the converter within each of the two feasible regions.

\section{A. Determining Switching Frequencies}

Using (25), it is possible to determine the output voltage of the converter under the FHA assumption [8], where $\eta$ is the efficiency of the converter assumed to be 0.9 .

$$
V_{o}=V_{s} \frac{\eta^{2}}{\sqrt{1+Q^{2}\left(\omega_{n}-\omega_{n}^{-1}\right)^{2}}}
$$

The highest and lowest frequencies needed to stay at $V_{o}$ are found in the corners of the perturbations i.e. when $V_{s}$ and $R_{L}$ are at either the maximum or minimum. By sweeping $\omega_{n}$, all obtainable output voltages for the converter can be determined. Fig. 8 shows the output voltage versus the normalized frequency for each corner. The dotted lines indicate the minimum $\omega_{n_{\text {min }}}$ and maximum $\omega_{n_{\max }}$ normalized frequency needed to stay at $V_{o}$ for the worst-case perturbations.

TABLE II: Design Criteria

\begin{tabular}{c|cl}
\hline & Value & Unit \\
\hline$V_{o}$ & 150 & $\mathrm{~V}$ \\
$V_{s}$ & $300 \pm 50$ & $\mathrm{~V}$ \\
$R_{L}$ & $1000 \pm 250$ & $\Omega$ \\
\hline
\end{tabular}




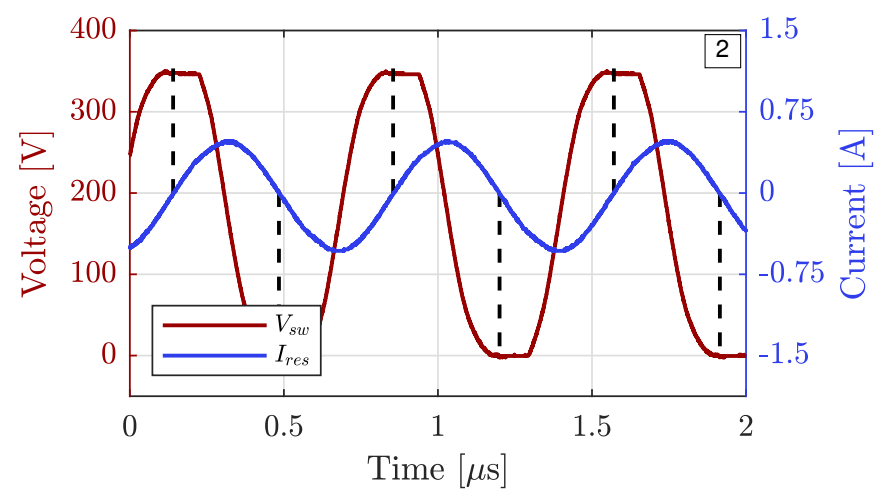

(a) Class-DE ZVS/ZVdS; $1.40 \mathrm{MHz}, t_{d}=263 \mathrm{~ns} / \mathrm{D}=13.2 \%$

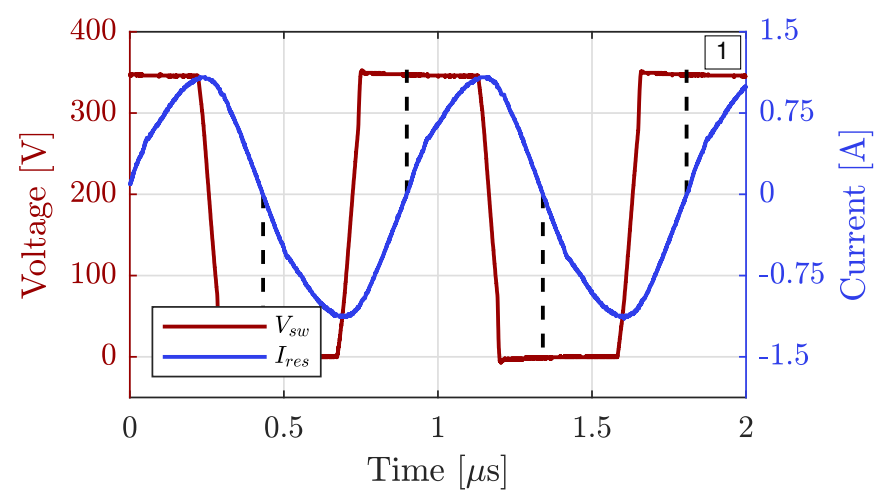

(c) Partial Hard-Switching; $1.10 \mathrm{MHz}, t_{d}=60 \mathrm{~ns} / \mathrm{D}=43.4 \%$

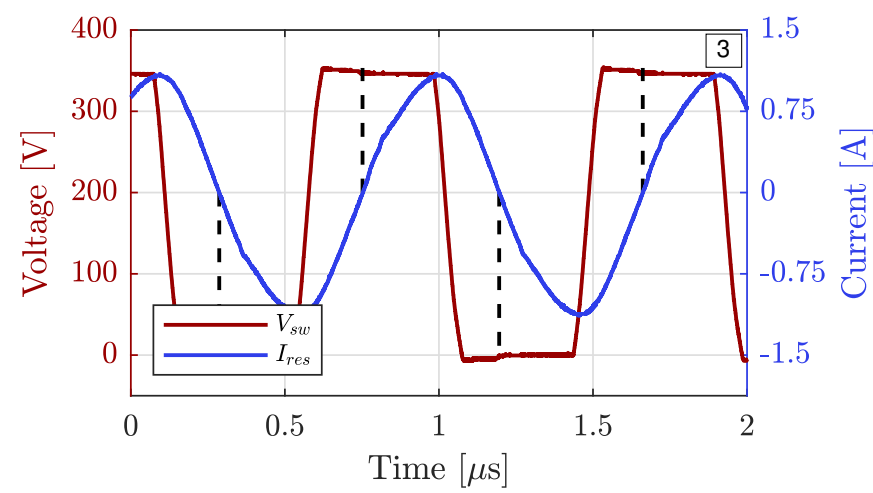

(e) Reverse Conduction; $1.10 \mathrm{MHz}, t_{d}=203 \mathrm{~ns} / \mathrm{D}=27.7 \%$

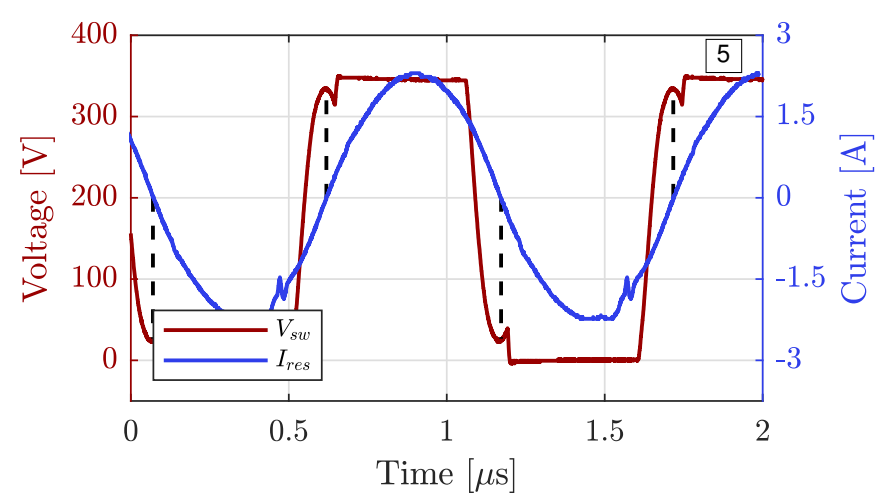

(g) Partial Hard-Switching w/ Current Reversal; $907 \mathrm{kHz}, t_{d}=139 \mathrm{~ns} /$ $D=37.4 \%$

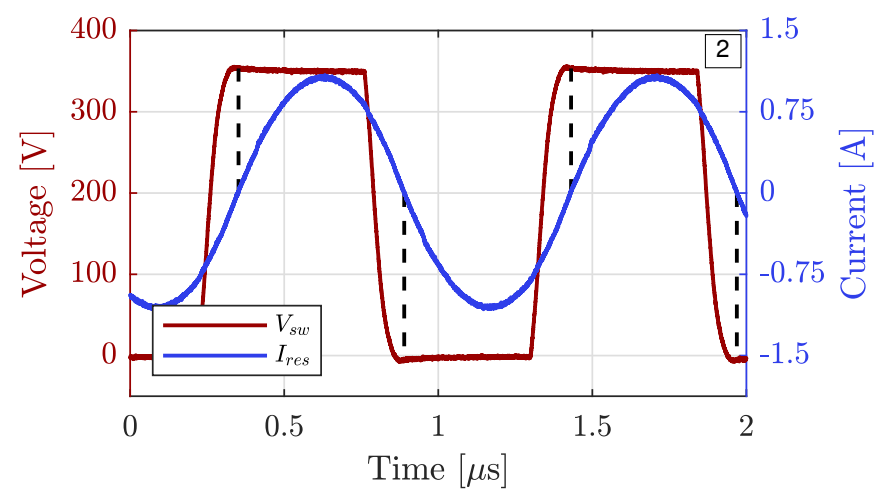

(b) Class-DE ZVS/ZVdS; $926 \mathrm{kHz}, t_{d}=130 \mathrm{~ns} / \mathrm{D}=38.0 \%$

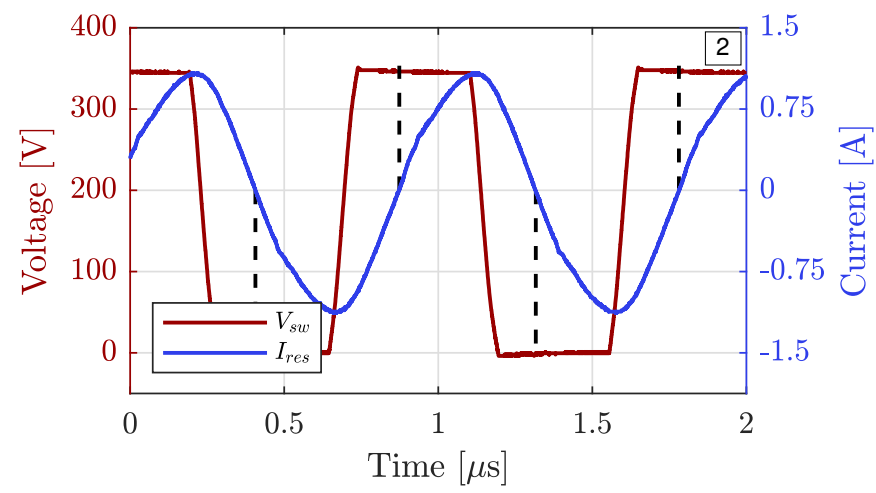

(d) ZVS; $1.1 \mathrm{MHz}, t_{d}=105 \mathrm{~ns} / \mathrm{D}=38.5 \%$

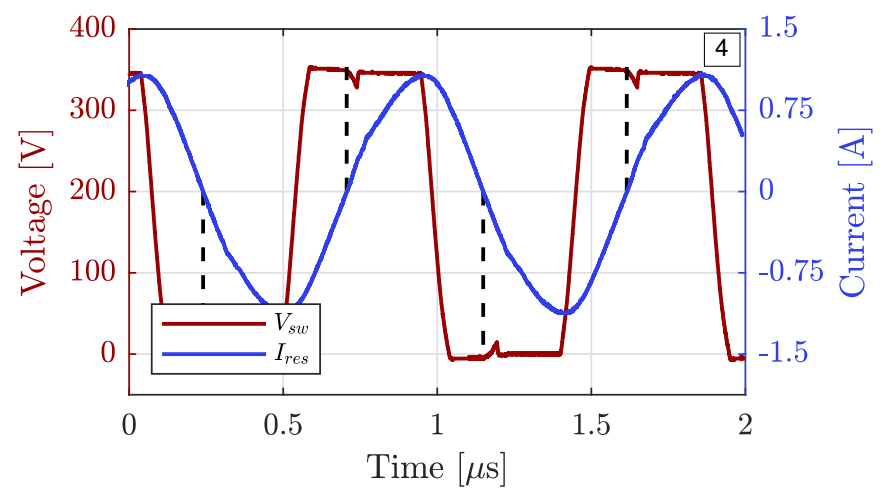

(f) Current Reversal; $1.10 \mathrm{MHz}, t_{d}=239 \mathrm{~ns} / \mathrm{D}=23.7 \%$

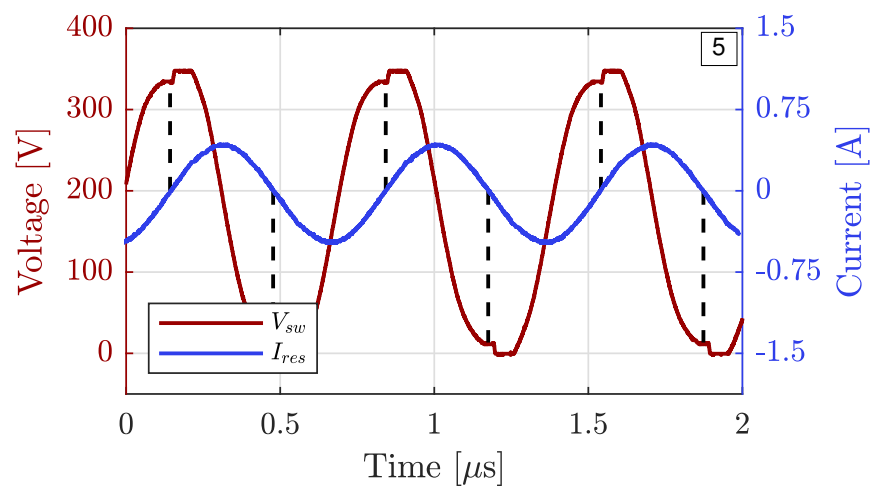

(h) Partial Hard-Switching w/ Current Reversal; $1.43 \mathrm{MHz}, t_{d}=287 \mathrm{~ns} /$ $D=9.0 \%$

Fig. 7: Captured waveforms of the switching node $V_{s w}$ and the resonant tank current $I_{r}$ for the different operating regions. Frequency, dead time and duty cycle are noted for each plot. The dashed lines indicate the zero-crossing of the current. 


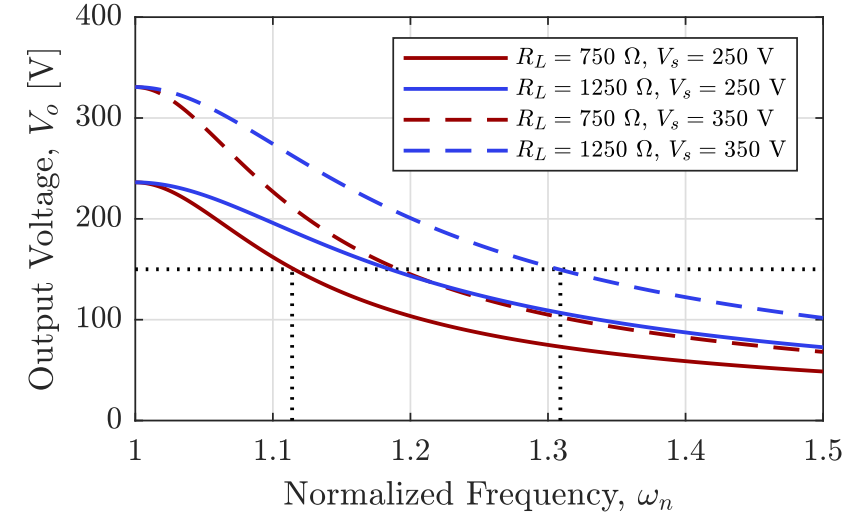

Fig. 8: Output voltage versus normalized frequency for each corner.

\section{B. Deriving Feasible Operation Regions and Dead Time}

In Section III-A, it is stated that the feasible operating region's only dependence on $V_{s}$ is through the nonlinear parasitic capacitance $C_{o s s}(t)$, and that within a local neighborhood of the nominal supply voltage, the capacitance change is negligible. From the design criteria in Table II we find that the supply varies with $\pm 50 \mathrm{~V}$ resulting in a $\mp 4.4 \mathrm{pF}$ variation in $C_{o s s(t r)}$. For the used converter and its defined load range, this variation in $C_{o s s(t r)}$ turns out to be small enough to be disregarded. Thus only $R_{L}$ needs to be considered when evaluating the feasible region.

Changing $R_{L}$ will either expand or contract the feasible operating region due to the corresponding change in the resonant tank current's amplitude. When the load is increased i.e. $R_{L}$ is decreased, the feasible operating region expands up and down in frequency and towards lower dead times while the current reversal limit stays somewhat stationary. The expansion happens because a larger current is being processed in the converter, which provides more current for faster charging/discharging $C_{o s s(t)}$. Hence less dead time is needed. When the load is decreased i.e. $R_{L}$ is increased, the opposite happens, and the region contracts because less current is available for charging/discharging $C_{o s s(t)}$. The change in the size of the feasible region, especially the contracting, vastly limits the size of realizable bounds on both $V_{s}$ and $R_{L}$ because the needed frequency range needs to fit within a smaller area. Fig. 9 shows the feasible operating regions for when the load is large and small.

The horizontal dotted lines on Fig. 9 indicate the frequency range that needs to be inside the feasible operating region. From Fig. 8, we know that $\omega_{n_{\max }}$ needs to be in the feasible region for $R_{L}=1250 \Omega$ and $\omega_{n_{\text {min }}}$ in the feasible region for $R_{L}=750 \Omega$. This results in a needed normalized dead time of 0.33 and 0.7 respectively. Since the normalized dead time of 0.7 works for both feasible operating regions, it will work for all bounded perturbations. Henceforth, the minimum dead time needed to meet the criteria in Table II is 0.7. Moreover, the found dead time is the smallest possible and the one that will exhibit the fewest losses in the converter while keeping

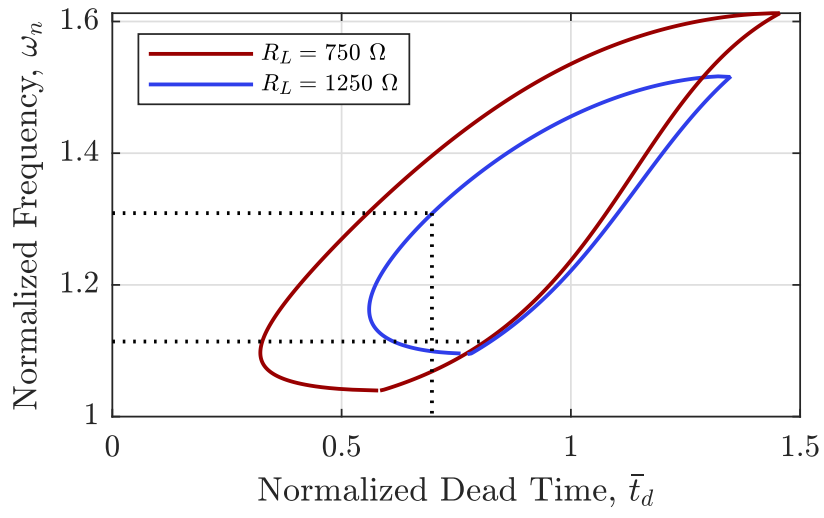

Fig. 9: The feasible operating region when the load is at the upper bound $\left(R_{L}=750 \Omega\right)$ and the lower bound $\left(R_{L}=\right.$ $1250 \Omega)$.

an FM-controlled converter inside the feasible region for all bounded perturbations. This is easily seen in Fig. 9 by the fact that the corner $\left(R_{L}=1250 \Omega\right)$ is a ZVS point.

\section{Validation}

To validate the just found results, the prototype is measured at each corner and the nominal case to verify that the output voltage is as expected and within the feasible region. First, the normalized results are converted to the proper units.

$$
\begin{gathered}
t_{d}=\bar{t}_{d} / \omega_{c}=128 \mathrm{~ns} \\
f_{s w_{\max }}=\frac{\omega_{n_{\max }} \omega_{c}}{2 \pi}=1.17 \mathrm{MHz} \\
f_{s w_{\min }}=\frac{\omega_{n_{\max }} \omega_{c}}{2 \pi}=0.963 \mathrm{MHz}
\end{gathered}
$$

Table III shows the measured output voltage for each of the corner cases and the nominal case, as well as the operating regions, when the dead time is $t_{d}=128 \mathrm{~ns}$. As predicted, the corner $\left(R_{L}=1250 \Omega, V_{s}=350 \mathrm{~V}\right)$ is a ZVS point, and all other corners experience reverse conduction losses, indicating they are within the feasible operating region. Finally, we see that all the measured points are close to the target output voltage $V_{o}=150 \mathrm{~V}$.

TABLE III: Measured supply, and output voltage for each corner and the nominal case when $t_{d}=128 \mathrm{~ns}$.

\begin{tabular}{ccc|cc|l}
\hline \multicolumn{3}{c|}{ Conditions } & \multicolumn{3}{c}{ Measured } \\
\hline$V_{s}[\mathrm{~V}]$ & $R_{L}[\Omega]$ & $f_{s w}[\mathrm{MHz}]$ & $V_{s}[\mathrm{~V}]$ & $V_{o}[\mathrm{~V}]$ & Operating Region \\
\hline 300 & 1000 & 1.05 & 300.1 & 149.7 & Rev. Conduction \\
250 & 1250 & 1.05 & 250.0 & 152.3 & Rev. Conduction \\
350 & 1250 & 1.17 & 350.9 & 146.6 & ZVS \\
250 & 750 & 0.96 & 250.4 & 148.5 & Rev. Conduction \\
350 & 750 & 1.03 & 350.6 & 144.4 & Rev. Conduction \\
\hline
\end{tabular}




\section{CONCLUSION}

This paper presents an analysis of the different switching loss modes in a class-D SRC using either a fixed dead time or fixed duty cycle. A feasible operating region where the FETs in the inverter stage only exhibit reverse conduction losses is defined and fully enclosed by the ZVS curve and the reverse current limit. We find that class-DE operation is possible at both the top and bottom of the feasible region where the ZVS curve intersects with the reverse current limit. Furthermore, the impact of using a fixed dead time compared to a fixed duty cycle is investigated. It is concluded that using a fixed dead time is superior to using a fixed duty cycle since a broader operating range can be achieved for the same reverse conduction losses, or the same operating range can be achieved with lower losses. A reduction in the reverse conduction losses of up to $59 \%$ or an expansion of the operating range by $33 \%$ when using a fixed dead time rather than a fixed duty cycle is calculated. The analysis and modeling approach is validated on a $1-\mathrm{MHz}$ class-D SRC prototype employing GaN switching devices, where the measurements followed the patterns predicted by the model. Lastly, we show how the presented analysis can be used to work out the frequency range and dead time for a frequency controller, such that the converter always stays within the feasible region, and the losses are minimized.

\section{REFERENCES}

[1] D. J. Perreault, J. Hu, J. M. Rivas, Y. Han, O. Leitermann, R. C. N. Pilawa-Podgurski, A. Sagneri, and C. R. Sullivan, "Opportunities and Challenges in Very High Frequency Power Conversion," in 2009 Twenty-Fourth Annual IEEE Applied Power Electronics Conference and Exposition, pp. 1-14, Feb 2009.

[2] A. Knott, T. M. Andersen, P. Kamby, J. A. Pedersen, M. P. Madsen, M. Kovacevic, and M. A. Andersen, "Evolution of very high frequency power supplies," IEEE Journal of Emerging and Selected Topics in Power Electronics, vol. 2, no. 3, pp. 386-394, 2014.

[3] J. M. Rivas, D. Jackson, O. Leitermann, A. D. Sagneri, Y. Han, and D. J. Perreault, "Design considerations for very high frequency dc-dc converters," in Power Electronics Specialists Conference, 2006. PESC'06. 37th IEEE, pp. 1-11, IEEE, 2006.

[4] N. Bertoni, G. Frattini, R. G. Massolini, F. Pareschi, R. Rovatti, and G. Setti, "An Analytical Approach for the Design of Class-E Resonant DC-DC Converters," IEEE Transactions on Power Electronics, vol. 31, no. 11, pp. 7701-7713, 2016.

[5] P. C. Luk, S. Aldhaher, W. Fei, and J. F. Whidborne, "State-Space Modeling of a Class $E^{2}$ Converter for Inductive Links," IEEE Transactions on Power Electronics, vol. 30, pp. 3242-3251, June 2015.

[6] X. Gao, H. Wu, and Y. Xing, "A Multioutput LLC Resonant Converter With Semi-Active Rectifiers," IEEE Journal of Emerging and Selected Topics in Power Electronics, vol. 5, no. 4, pp. 1819-1827, 2017.

[7] M. Noah, S. Endo, H. Ishibashi, K. Nanamori, J. Imaoka, K. Umetani, and M. Yamamoto, "A Current Sharing
Method Utilizing Single Balancing Transformer for a Multiphase LLC Resonant Converter With Integrated Magnetics," IEEE Journal of Emerging and Selected Topics in Power Electronics, vol. 6, no. 2, pp. 977-992, 2018.

[8] M. K. Kazimierczuk and D. Czarkowski, Resonant power converters. John Wiley \& Sons, 2012.

[9] X. Wei, H. Sekiya, T. Nagashima, M. K. Kazimierczuk, and T. Suetsugu, "Steady-state analysis and design of class-d zvs inverter at any duty ratio," IEEE Transactions on Power Electronics, vol. 31, no. 1, pp. 394-405, 2015.

[10] Y. Nagata, Y. Yamada, Y. Fukumoto, T. Ikenari, X. Wei, T. Suetsugu, and H. Sekiya, "The phase-controlled classd zvs inverter with current protection," in 2017 IEEE Energy Conversion Congress and Exposition (ECCE), pp. 2176-2183, IEEE, 2017.

[11] F. M. Spliid, A. M. Ammar, and A. Knott, "Analysis and design of a resonant power converter with wide input voltage range for ac/dc applications," IEEE Journal of Emerging and Selected Topics in Power Electronics, 2019

[12] A. M. Ammar, F. M. Spliid, Y. Nour, and A. Knott, "Analysis and design of a charge-pump-based resonant acdc converter with inherent pfc capability," IEEE Journal of Emerging and Selected Topics in Power Electronics, 2020.

[13] M. F. Menke, Á. R. Seidel, and R. V. Tambara, "LLC LED Driver Small-Signal Modeling and Digital Control Design for Active Ripple Compensation," IEEE Transactions on Industrial Electronics, vol. 66, no. 1, pp. 387-396, 2019.

[14] G. Chen, H. Li, X. Sun, J. Zhou, and L. Bai, "Analysis and design of llc converter based on sic mosfet," in 2018 13th IEEE Conference on Industrial Electronics and Applications (ICIEA), pp. 1443-1448, IEEE, 2018.

[15] H. Wen, J. Gong, X. Zhao, C.-S. Yeh, and J.-S. Lai, "Analysis of diode reverse recovery effect on zvs condition for gan-based llc resonant converter," IEEE Transactions on Power Electronics, vol. 34, no. 12, pp. 11952-11963, 2019.

[16] A. M. Ammar, Y. Nour, and A. Knott, "A high-efficiency 1 mhz 65 w gan-based llc resonant dc-dc converter," in 2019 IEEE Conference on Power Electronics and Renewable Energy (CPERE), pp. 448-452, IEEE, 2019.

[17] H. Ma, Y. Li, Q. Chen, L. Zhang, and J. Xu, "A Single-Stage Integrated Boost-LLC AC-DC Converter With Quasi-Constant Bus Voltage for Multichannel LED Street-Lighting Applications," IEEE Journal of Emerging and Selected Topics in Power Electronics, vol. 6, no. 3, pp. 1143-1153, 2018.

[18] S. Saito, S. Mita, W. Zhu, H. Onishi, S. Nagaoka, T. Uematsu, K. Nguyen, and H. Sekiya, "Novel design approach of soft-switching resonant converter with performance visualization algorithm," IEEE Access, vol. 8, pp. 59922 59933, 2020.

[19] T. Nagashima, X. Wei, T. Suetsugu, M. K. Kazimierczuk, and H. Sekiya, "Waveform equations, output power, and power conversion efficiency for class-e inverter outside nominal operation," IEEE Transactions on Industrial Electronics, vol. 61, no. 4, pp. 1799-1810, 2013. 
[20] B. Guo, S. Dwari, and S. Priya, "Voltage-controlled tunable capacitor based resonant power converter," in 2019 IEEE Energy Conversion Congress and Exposition (ECCE), pp. 2164-2169, IEEE, 2019.

[21] Y. Hu, A. Amara, and A. Ioinovici, "Llc resonant converter operated at constant switching frequency and controlled by means of a switched-capacitor circuit," in 2013 1st International Future Energy Electronics Conference (IFEEC), pp. 691-696, IEEE, 2013.

[22] K. Harada, A. Katsuki, M. Fujiwara, H. Nakajima, and H. Matsushita, "Resonant converter controlled by variable capacitance devices," IEEE Transactions on Power Electronics, vol. 8, no. 4, pp. 404-410, 1993.

[23] I. Kolberg, D. Shmilovitz, and S. S. Ben-Yaakov, "Ceramic capacitor controlled resonant llc converters," in 2018 IEEE Applied Power Electronics Conference and Exposition (APEC), pp. 2162-2167, IEEE, 2018.

[24] K.-H. Lee and J.-I. Ha, "Resonant switching cell model for high-frequency single-ended resonant converters," IEEE Transactions on Power Electronics, vol. 34, no. 12, pp. 11897-11911, 2019.

[25] W. Zhong and S. Hui, "Maximum energy efficiency operation of series-series resonant wireless power transfer systems using on-off keying modulation," IEEE Transactions on Power Electronics, vol. 33, no. 4, pp. 3595-3603, 2017.

[26] K.-H. Lee, E. Chung, Y. Han, and J.-I. Ha, “A family of high-frequency single-switch dc-dc converters with low switch voltage stress based on impedance networks," IEEE Transactions on Power Electronics, vol. 32, no. 4, pp. 2913-2924, 2016.

[27] J. M. Rivas, O. Leitermann, Y. Han, and D. J. Perreault, "A very high frequency $\mathrm{dc}-\mathrm{dc}$ converter based on a class $\phi 2$ resonant inverter," IEEE Transactions on Power Electronics, vol. 26, no. 10, pp. 2980-2992, 2011.

[28] N. J. Dahl, A. M. Ammar, A. Knott, and M. A. Andersen, "An improved linear model for high frequency classde resonant converter using the generalized averaging modeling technique," IEEE Journal of Emerging and Selected Topics in Power Electronics, 2019.

[29] M. Salem, V. K. Ramachandaramurthy, A. Jusoh, S. Padmanaban, M. Kamarol, J. Teh, and D. Ishak, "Threephase series resonant dc-dc boost converter with double llc resonant tanks and variable frequency control," IEEE Access, vol. 8, pp. 22386-22399, 2020.

[30] M. Salem, A. Jusoh, N. R. N. Idris, C. W. Tan, and I. Alhamrouni, "Phase-shifted series resonant dc-dc converter for wide load variations using variable frequency control," in 2017 IEEE Conference on Energy Conversion (CENCON), pp. 329-333, IEEE, 2017.

[31] S. Zou, A. Mallik, J. Lu, and A. Khaligh, "Sliding mode control scheme for a cllc resonant converter," IEEE Transactions on Power Electronics, vol. 34, no. 12, pp. 12274-12284, 2019.

[32] T. Osato, X. Wei, K. Nguyen, H. Sekiya, et al., "Steadystate analysis and design of phase-controlled class-d zvs inverter," Nonlinear Theory and Its Applications, IEICE, vol. 11, no. 2, pp. 189-205, 2020.
[33] Y. Yamada, T. Nagashima, Y. Ibuki, Y. Fukumoto, T. Ikenari, and H. Sekiya, "Design of a dc-dc converter with phase-controlled class-d zvs inverter," IEEE Journal on Emerging and Selected Topics in Circuits and Systems, vol. 5, no. 3, pp. 354-363, 2015.

[34] M. Ekhtiari, T. Andersen, M. A. Andersen, and Z. Zhang, "Dynamic optimum dead time in piezoelectric transformerbased switch-mode power supplies," IEEE Transactions on Power Electronics, vol. 32, no. 1, pp. 783-793, 2016

[35] D. C. Hamill, "Class de inverters and rectifiers for dc-dc conversion," in PESC Record. 27th Annual IEEE Power Electronics Specialists Conference, vol. 1, pp. 854-860, IEEE, 1996.

[36] GaNSystems, “GaN transistor GS66502B,” 2017.

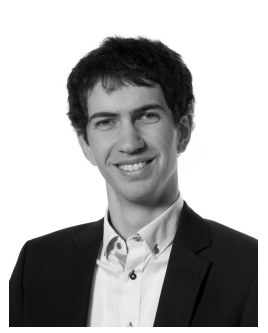

Nicolai J. Dahl (S'19) is a Ph.D. student at the electronics group at the Technical University of Denmark, Kongens Lyngby, Denmark. He received both his B.Sc. degree and M.Sc. degree from the Technical University of Denmark in June 2016 and August 2018 respectively. His research interest include control theory, system modeling, signal processing, and optimization, which he has applied in the fields of switch-mode power amplifiers, resonant converters, and his current research topic, time-based control.

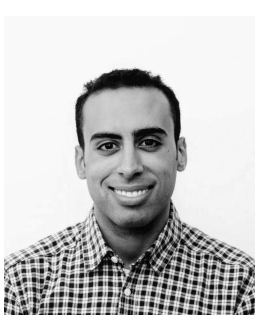

Ahmed M. Ammar ( ${ }^{\prime}$ 11, M' 20) received the B.Sc. degree in electronics and communications engineering from Mansoura University, Mansoura, Egypt, in 2011, the M.Sc. degree in microelectronics system design from Nile University, Giza, Egypt, in 2014, and the Ph.D. degree in electrical engineering from the Technical University of Denmark, Kongens Lyngby, Denmark, in 2020. His experience includes positions at different industries, including Intel Corporation, Hillsboro, OR, USA, Imec, Leuven, Belgium, and Mentor Graphics, Cairo, Egypt (now Mentor, a Siemens Business). He was a visiting researcher at the University of Minnesota, Minneapolis, USA, and the University of Oxford, Oxford, UK. He is currently a postdoc researcher in the Department of Electrical Engineering, Technical University of Denmark. His current research interests include power electronics, integrated power converters, and power management and delivery circuits and systems.

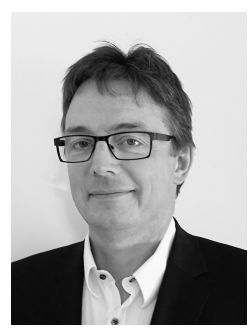

Michael A. E. Andersen (M'88) received the M.Sc.E.E. and Ph.D. degrees in power electronics from the Technical University of Denmark, Kongens Lyngby, Denmark, in 1987 and 1990, respectively. $\mathrm{He}$ is currently a Professor of power electronics at the Technical University of Denmark, where since 2009, he has been the Deputy Head of the Department of Electrical Engineering. He is the author or coauthor of more than 300 publications. His research interests include switch-mode power supplies, piezoelectric transformers, power factor correction, and switchmode audio power amplifiers. 